\title{
Top quark production at ATLAS
}

\author{
Naoki Kimura On behalf of the ATLAS Collaboration \\ Waseda University, 3-4-1 Okubo, Shinjuku, Tokyo
}

\begin{abstract}
We present various studies of the production of top quarks in proton-proton collisions at 7 TeV with the ATLAS detector at the Large Hadron Collider. The cross-section of top-quark pair production is measured in the single lepton, dilepton, all hadronic channel and channels with tau leptons. Top-quark pairs are also studied in the context of additional radiation. Measurements of single top-quark production in the t-channel and Wt-channel are also presented. Several extensions of the SM predict the presence of new particles that couple to top quarks. We report on searches for such resonance decaying to top-antitop quark pairs.
\end{abstract}

Keywords: ATLAS, top, production

\section{Introduction}

In 2011, the LHC delivered an integrated luminosity of $\mathcal{L}_{\text {int }}=5.6 \mathrm{fb}^{-1}$ of $p p$ collisions at $7 \mathrm{TeV}$ center-of-mass energy to the ATLAS [1] experiment. The data fulfilling all quality requirements of top quark analysis corresponds to the integrated luminosity of $\mathcal{L}_{\text {int }}=4.7 \mathrm{fb}^{-1}$. Discovered in 1995 [2, 3], the top quark is the heaviest known elementary particle. The precision measurement of the top quark production cross-section and study of top quark properties provides a good test of perturbative QCD, and is also an important variable to observe deviations from Standard Model predictions, that could indicate new physics.

At the LHC top quarks are predominantly produced via the strong interaction processes resulting in pair production of top and antitop, the theoretical cross-section being calculated to be $\sigma_{t \bar{t}}=167_{-18}^{+17} \mathrm{pb}$ [4]. The weak interaction production leading to single top quark final states includes the exchange of a virtual $\mathrm{W}$ boson in the $t$-channel or in the $s$-channel, and the associated production of a top quark and an on-shell $\mathrm{W}$ boson. Calculated cross-section are $\sigma_{t}=64.6_{-2.0}^{+2.7} \mathrm{pb}[5], \sigma_{s}=4.6 \pm 0.2$ $\mathrm{pb}[6], \sigma_{W t}=15.7 \pm 1.1 \mathrm{pb}$ [7] respectively. The lifetime of the top quark is shorter than the hadronisation time scale, thus it decays almost $100 \%$ to a $\mathrm{W}$ boson and a b-quark keeping top quark spin information. The

\footnotetext{
* Speaker

Email address: naoki.kimura@cern.ch (Naoki Kimura On behalf of the ATLAS Collaboration)

Preprint submitted to Nuc. Phys. (Proc. Suppl.)
}

final decay topologies are determined by the decay of the $\mathrm{W}$ boson, either into a lepton-neutrino pair, or into a pair of quarks.

\section{Top quark pair production cross-section}

In the lepton+jets channel (branching ratio $\mathrm{BR}=$ $34 \%$ ), events are selected with exactly one lepton (electron $(e)$ or muon $(\mu))$, at least three jets and large missing transverse momentum $\left(E_{T}^{\text {miss }}\right)$. The main background is $\mathrm{W}+$ jets events. Kinematic variables are selected to distinguish between the $t \bar{t}$ signal and background, and a likelihood discriminant is constructed. The crosssection is extracted from a maximum likelihood fit to the discriminant distributions. The measured cross-section using $0.7 \mathrm{fb}^{-1}[8]$ is

$$
\sigma_{t \bar{t}}=179.0 \pm 3.9(\text { stat } .) \pm 9.0(\text { syst } .) \pm 6.6(\text { lumi } .) \mathrm{pb} .
$$

Main systematic uncertainties are from the choice of the signal Monte Carlo(MC) generator, Jet Energy Scale (JES) and initial final state radiation modeling (ISR/FSR).

Dilepton channel $(e e, \mu \mu, e \mu)(\mathrm{BR}=5 \%)$ are required two oppositely-charged leptons, and $E_{T}^{\text {miss }}$ and at least two jets. The main background are Drell-Yan and $\mathrm{W}+$ jets events. The cross-section is measured using a cut-based analysis with a profile likelihood fit to the number of observed events. The measured cross-section using $0.7 \mathrm{fb}^{-1}[9]$ is

$$
\sigma_{t \bar{t}}=176 \pm 5(\text { stat } .)_{-11}^{+14}(\text { syst. }) \pm 8(\text { lumi. }) \mathrm{pb} .
$$

August 31, 2012 
Main systematic uncertainties are from signal MC generator and Jet $/ E_{T}^{\text {miss }}$ modeling.

The measurement of $\sigma_{t \bar{t}}$ in the all-hadronic channel has the advantage of a large $B R=44 \%$ but suffers from huge QCD background. Events are selected with at least six jets where two are $b$-tagged and events with isolated leptons are vetoed. The cross-section is estimated by kinematic fit to reconstructed the top quark mass. The measured cross-section using $4.7 \mathrm{fb}^{-1}[10]$ is

$$
\sigma_{t \bar{t}}=168 \pm 12(\text { stat } .)_{-57}^{+60}(\text { syst. }) \pm 7(\text { lumi } .) \mathrm{pb} .
$$

Main systematic uncertainties are from JES, $b$-tag efficiency and ISR/FSR.

A combination of $\sigma_{t \bar{t}}$ measurements [11] is presented in the lepton+Jets and dilepton channels, both using $0.7 \mathrm{fb}^{-1}$ of data, and the all-hadronic channel using $1.02 \mathrm{fb}^{-1}$. Hadronically decaying tau lepton and jet or lepton $(e \mu)$ channel covers the rest of $t \bar{t}$ decay. In hadronic tau + lepton channel, events are selected with exactly one lepton, at least two jets, $E_{T}^{\text {miss }}, b$-tagged jets and tau candidate. The cross-section is measured from multivariate discriminant. In hadronically decaying tau lepton and jet channel, events are selected with at least 5 jets, $2 b$-tagged jets, lepton veto and hadronic tau candidate. The cross-section is measured from fit to a characteristic track multiplicity distribution for the tau candidate. The individual and combined cross-section measurements are shown on the upper part of Figure 1 while all-hadronic channel using $4.7 \mathrm{fb}^{-1}$ data and result from events containing tau candidate are shown on the bottom part.

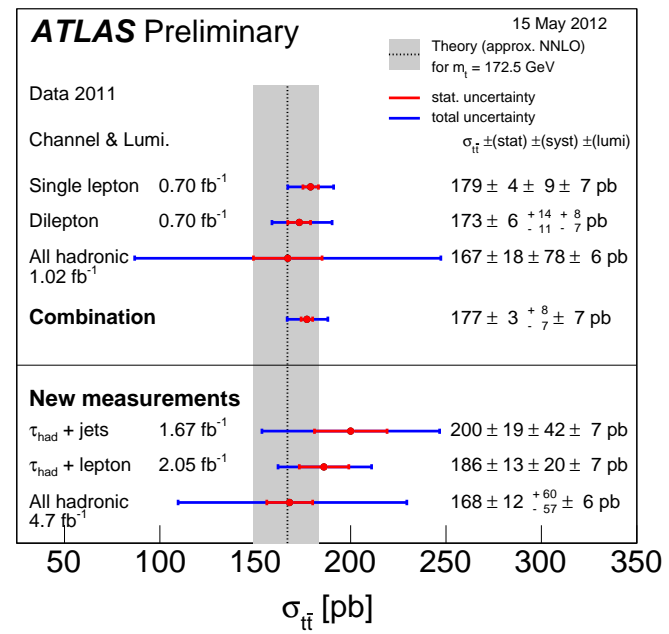

Figure 1: summary of ATLAS measurements of the $t \bar{t}$ production cross-section compared to the theoretical expectation

\section{Inclusive $t \bar{t} \gamma$ cross-section}

Cross-section of top quark pair with additional photons channel has a good sensitivity to the measurement of the $t \bar{t} \gamma$ vertex. A first measurement of the $t \bar{t} \gamma$ crosssection was performed using $1.04 \mathrm{fb}^{-1}$ of data. Events are selected with one lepton, at least 4 jets with at least one b-tagged jet, $E_{T}^{\text {miss }}$ and one photon. The crosssection is measured from fit to the track isolation distribution for the photon. Measured cross-section times branching ratio into the single and dilepton channel [12] is

$$
\sigma_{t \bar{t} \gamma} \times B R=2.0 \pm 0.5(\text { stat. }) \pm 0.7(\text { syst. }) \pm 0.08(\text { lumi. }) \text { pb. }
$$

Result is consistent with the theoretical prediction $2.1 \pm$ $0.4 \mathrm{pb}[13]$.

\section{4. $t \bar{t}$ production with a veto on additional central jet activity}

A measurement is presented of the jet activity arising from QCD emission from $t \bar{t}$ events using $2.05 \mathrm{fb}^{-1}$ of data in the dilepton channel [14]. Events are selected with two opposite sign leptons $(e$ or $\mu), E_{T}^{m i s s}$ and two $b$-tagged jets. A veto is then applied to events which contain an additional jet with a $p_{T}$ above a defined threshold in a central rapidity region. The measurement of jet activity is presented as the fraction of events surviving the jet veto, known as the gap fraction defined as $f\left(Q_{0}\right)=\frac{n\left(Q_{0}\right)}{N} \equiv \frac{\sigma\left(Q_{0}\right)}{\sigma}$ where $\mathrm{N}$ is the number of selected $t \bar{t}$ events passing selection, $n\left(Q_{0}\right)$ is the subset of these events that do not contain an additional jet with a $p_{T}>Q_{0}, \sigma$ is the fiducial cross-section for inclusive $t \bar{t}$ production, and $\sigma\left(Q_{0}\right)$ is the fiducial crosssection for events passing the jet veto. The measured $f\left(Q_{0}\right)$ from data is compared to the predictions from the ACERMC [15] generator with varied PYTHIA [16] parton shower parameters, and is shown in Figure 2. As a results of this measurement ATLAS has updated it's ISR systematic prescriptions, which will lead to all future ISR systematic uncertainties associated with future measurements being approximately $50 \%$ smaller.

\section{Single top quark production cross-section}

Single top production is a sensitive probe of a number of new physics model and it can also be used for direct measurements of the CKM matrix element $\left|V_{t b}\right|$. The cross-section measurements have been performed in the $t-$ [17], Wt- [18] and $s$-channels [19]. The measured values and their associated statistical and systematic uncertainties or upper observed and expected 


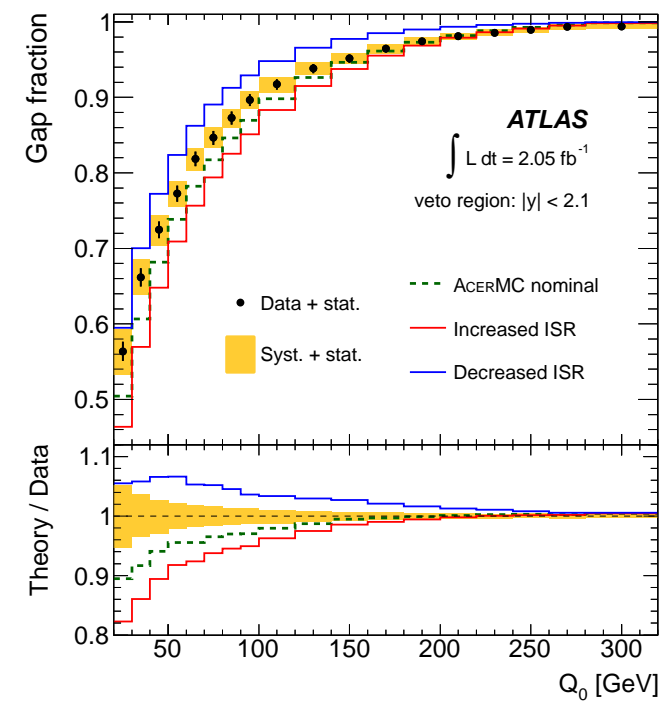

Figure 2: The measured $f\left(Q_{0}\right)$ compared with the ACERMC prediction increased and decreased ISR

Table 1: Summary of single top cross-section measurements.

\begin{tabular}{|c|c|c|}
\hline channel & $\begin{array}{l}\mathcal{L}_{\text {int }} \\
{\left[\mathrm{fb}^{-1}\right]}\end{array}$ & $\begin{array}{l}\text { measured (prediction) } \\
{[\mathrm{pb}]}\end{array}$ \\
\hline $\mathrm{t}$ & 1.04 & $\begin{array}{lcc}83 & \pm & 4(\text { stat } .)_{-19}^{+20}(\text { syst }) \\
\left(64.57_{-2.0}^{+2.7}\right) & \end{array}$ \\
\hline Wt & 2.05 & $\begin{array}{l}16.8 \pm 2.9 \text { (stat }) \pm 4.9 \text { (syst }) \\
(15.7 \pm 1.1)\end{array}$ \\
\hline $\mathrm{s}$ & 0.70 & $<26.5 @ 95 \% C L$ \\
\hline
\end{tabular}

95\% CL limits are reported in Table 1 All results are consistent with the SM prediction. The CKM matrix element $\left|V_{t b}\right|$ is measured to be $\left|V_{t b}\right|=1.13_{-0.13}^{+0.14}$ and $\left|V_{t b}\right|=1.03_{-0.19}^{+0.16}$ in $t$ - and $W t$-channel respectively. A search for the flavor-changing neutral currents (FCNCs) has also been performed for the single top production using $2.05 \mathrm{fb}^{-1}$ data [20]. the search results are consistent with the SM hypothesis and the following 95\% CL limits are set to $\sigma(q g \rightarrow t) \times B R(t \rightarrow W b)<3.9 p b$ and $B R(t \rightarrow u g)<5.7 \times 10^{-5}, B R(t \rightarrow c g)<2.7 \times 10^{-4}$.

\section{Charge Asymmetry}

Due to the asymmetry in the production via $q \bar{q}$ and qg, QCD predicts at the LHC a small excess of centrally produced antitop quark while top quarks are produced, on average, at higher absolute rapidities. Measurement of the top-antitop production charge asymmetry $\mathrm{A}_{C}$ has been performed using $1.04 \mathrm{fb}^{-1}$ data [21]. $\mathrm{A}_{C}$ is defined as

$$
A_{C}=\frac{N(\Delta|y|>0)-N(\Delta|y|<0)}{N(\Delta|y|>0)+N(\Delta|y|<0)}
$$

where $\Delta|y| \equiv\left|y_{t}\right|-\left|y_{t}\right|$ is the difference between the absolute values of the top and antitop rapidities and $N$ is the number of events with $\Delta|y|$ positive or negative. Events are selected with one lepton, $E_{T}^{\text {miss }}$ and at least four jets with at least one $b$-tagged jets. A kinematic fit was used to reconstruct the $t \bar{t}$ event topology. A Bayesian unfolding procedure was performed to correct for acceptance and detector effects. Measurement $\mathrm{A}_{C}$ is

$$
A_{C}=-0.018 \pm 0.028(\text { stat } .) \pm 0.023(\text { syst. })
$$

consistent with the prediction from the MC@NLO [22] Monte Carlo generator of $A_{C}=0006 \pm 0.002$.

\section{Top spin correlation}

A measurement of the spin correlation in $t \bar{t}$ production was performed using $2.1 \mathrm{fb}^{-1}$ [23]. The lifetime of the top quark is at least order of magnitude shorter than the timescale for strong interactions, implying that the top quark decays before hadronisation. Thus the spin of the top quark at production is transferred to its decay products and can be measured directory via their angular distributions. In this analysis, candidate events were selected in the dilepton topology with large $E_{T}^{\text {miss }}$ and at least two jets. the difference in azimuthal angle between leptons in the laboratory frame was used to extract the correlation between the top and antitop quark spins. In the helicity basis the measured degree of correlation corresponds to

$$
A_{\text {helicity }}=0.40 \pm 0.04(\text { stat } .)_{-0.07}^{+0.08}(\text { syst. })
$$

in agreement with the NLO SM prediction. The azimuthal angle between leptons distribution shown in Figure 3 is found to be a sensitive observable. The hypothesis of zero spin correlation is excluded at 5.1 standard deviations.

\section{Conclusions}

The knowledge of top-physics is extended by new and more precise results obtained at ATLAS with the data collected in 2011 at $7 \mathrm{TeV}$ centre-of-mass energy. All of the measurements are consistent with the SM predictions. It is expected that more data will be collected at $8 \mathrm{TeV}$ center-of-mass collisions during 2012 than in 2011 , hence, the statistical uncertainty of top quark 


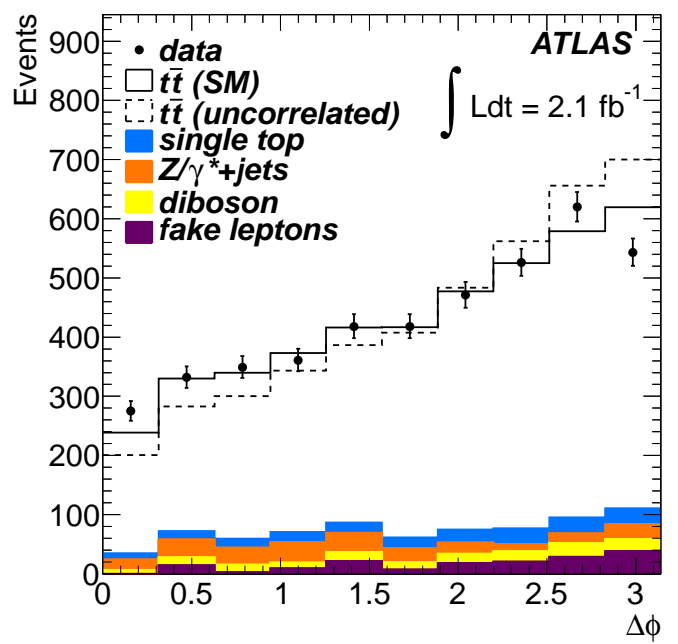

Figure 3: Azimuthal angle between leptons distribution used for the measurement of the spin correlation in the $t \bar{t}$ production.

measurements will be reduced. The systematic uncertainty due to the jet energy scale and generator modeling which are the main uncertainty sources in many measurements will also be improved due to the generator tuning and comparisons to the LHC data.

\section{References}

[1] ATLAS Collaboration, JINST 3, (2008) S08003.

[2] CDF Collaboration, F.Abe et al., Phys. Rev. Lett. 74 (1995) 2626-2631, arXiv/9503002.

[3] D0 Collaboration, S. Abachi et al., Phys. Rev. Lett. 74 (1995) 2632-2637, arXiv/9503003.

[4] M. Aliev et al., HAdronic Top and Heavy quarks crOss section calculatoR, arXiv:1007;1327 (2011).

[5] N. Kidonakis, Phys. Rev. D 83, 091503 (2011).

[6] N. Kidonakis, Phys. Rev. D 82, 054018 (2010).

[7] N. Kidonakis, Phys. Rev. D 81, 054028 (2010).

[8] ATLAS Collaboration, ATLAS-CONF-2011-121, http://cdsweb.cern.ch/record/1376413.

[9] ATLAS Collaboration, JHEP 1205 (2012)059, arXiv: 1202.4892

[10] ATLAS Collaboration, ATLAS-CONF-2012-031, https://cdsweb.cern.ch/record/1277665.

[11] ATLAS Collaboration, ATLAS-CONF-2012-024, https://cdsweb.cern.ch/record/1420935.

[12] ATLAS Collaboration, ATLAS-CONF-2011-153, https://cdsweb.cern.ch/record/1398197.

[13] K. Melnikov, M. Schulze and A. Scharf, Phys. Rev. D83 (2011) 074013 arXiv:1102.1967.

[14] ATLAS Collaboration, Eur.Phys.J C72 (2012) 2043, arXiv: 1203.5015 .

[15] B. Kersevan and E. Richter-Was, arXiv:hep-ph/0405247.

[16] T. Sostrand, S. Mrenna, P. Skands, JHEP 05 (2006) 026.

[17] ATLAS Collaboration, submitted to Phys. Lett. B, arXiv: 1205.3130.

[18] ATLAS Collaboration, submitted to Phys. Lett. B, arXiv:1205.5764.
[19] ATLAS Collaboration, ATLAS-CONF-2011-118, http://cdsweb.cern.ch/record/1376410.

[20] ATLAS Collaboration, Phys. Lett. B 712, (2012) 351-369.

[21] ATLAS Collaboration, submitted to Eur. Phys. J. C, arXiv/1203.4211.

[22] S. Frixione and B.R. Webber, JHEP 0206 (2002) 029 arXiv:0204244.

[23] ATLAS Collaboration, Phys. Rev. Lett. 108, 212001 (2012) 